\title{
Synergy in B-Cell Activation between Toll-Like Receptor 9 and Transmembrane Activator and Calcium-Modulating Cyclophilin Ligand Interactor (TACI) in A181E/C104R Compound Heterozygous Siblings
}

\author{
Annick A. J. M. van de Ven, ${ }^{1}$ Willemijn J. M. Janssen, ${ }^{1}$ Lisette van de Corput, ${ }^{2}$ \\ Andries C. Bloem, ${ }^{2}$ Joris M. van Montfrans, ${ }^{1}$ and Marianne Boes ${ }^{1}$ \\ ${ }^{1}$ Department of Pediatric Immunology and Infectious Diseases, Wilhelmina Children's Hospital, University Medical Center Utrecht, \\ Lundlaan 6, P.O. Box 85090, KC.01.069.0, 3584 EA Utrecht, The Netherlands \\ ${ }^{2}$ Department of Medical Immunology, University Medical Center Utrecht, Heidelberglaan 100, F03.821, P.O. Box 85500, \\ 3508 GA Utrecht, The Netherlands
}

Correspondence should be addressed to Marianne Boes; mboes@umcutrecht.nl

Received 21 April 2013; Accepted 26 May 2013

Academic Editors: S.-i. Fujii, F. Granucci, W. W. Leitner, and S. Sánchez-Ramón

Copyright (C) 2013 Annick A. J. M. van de Ven et al. This is an open access article distributed under the Creative Commons Attribution License, which permits unrestricted use, distribution, and reproduction in any medium, provided the original work is properly cited.

\begin{abstract}
Purpose. Approximately 9\% of common variable immunodeficiency (CVID) patients harbor variants in the transmembrane activator and CAML interactor gene, TACI, which contribute to CVID development. We found identical compound heterozygous TACI variants (C104R and A181E) in kindred of which one sibling had severe CVID with refractory auto immunity, and a second sibling remained asymptomatic. This study investigated possible differences in B-cell phenotype and function that could explain this divergent clinical expression. Methods. C104R and A181E TACI variants were identified through Sanger sequencing. Phenotypic evaluation of the lymphocyte compartment was performed by flow cytometry analyses. Lymphoblastoid cell lines (LCL) from the index patient, asymptomatic sibling, and controls were generated. Intracellular TACI expression was determined, and activationassociated calcium flux capacity was measured. In vitro stimulation assays and RT PCR were performed. Results. Both intracellular levels and surface expressed TACI protein were higher in the asymptomatic sibling than the CVID patient as were TACItriggering-induced mRNA expression AID and production of Ig class-switched antibodies. In analogy, the asymptomatic sibling displayed enhanced Toll-like receptor 9 expression and signaling, suggesting a compensatory immune mechanism. Conclusions. Posttranscriptional regulation of TACI protein and cross-talk with TLR9 signaling may contribute to phenotypic diversity between individuals with TACI variants.
\end{abstract}

\section{Introduction}

Common variable immunodeficiency (CVID) is a primary immunodeficiency characterized by recurrent bacterial infections, hypogammaglobulinemia, and impaired antigenspecific antibody synthesis [1]. In approximately $10 \%$ of patients, a genetic defect has been identified. In addition, functional defects have been described, for example, in Toll-like receptor (TLR) signaling $[2,3]$. The most prevalent genetic alterations are located in the TNFRSF13B gene encoding transmembrane activator, calcium modulator, and cyclophilin ligand (CAML) interactor (TACI) [4]. TACI is mainly expressed on B-cells and belongs to the tumor necrosis factor receptor (TNFR) family [4].

Two ligands for TACI have been described: B-cell activating factor (BAFF) [5] and a proliferation-inducing ligand (APRIL) $[6,7]$. In addition, TACI may bind proteoglycans including syndecan-2 that stimulate TACI-mediated signaling $[8,9]$. BAFF and APRIL also bind B-cell maturation antigen (BCMA) [10], and BAFF has a third receptor, BAFFR [11]. 
This versatility complicates studying the precise contribution of each molecule to humoral immunity [9].

Following ligand binding, the intracellular TACI domains interact with TNFR-associated proteins (TRAFs) and CAML. CAML interaction activates nuclear factor of activated $\mathrm{T}$ cells (NFAT), which has a key role in controlling calcium release from intracellular stores [4]. Additionally, nuclear factor kappa $\mathrm{B}(\mathrm{NF} \kappa \mathrm{B})$ is activated [12], probably via TRAFs, c-Jun NH2-terminal kinase, and transcription factor AP-1 [4]. TACI has a role in maintenance of B-cell homeostasis, immunoglobulin (Ig) isotype class-switching, and antibody production in response to type II T-cell-independent antigens. Furthermore it may act in synergy with CD40 and TLRs [13-15]. It was recently shown that TACI induces classswitch recombination via the myeloid differentiation primary response gene 88 (MyD88) adaptor, thus employing the same signaling cascade as multiple TLRs [16].

Currently, TACI is considered a CVID susceptibility gene (relative risk 4.3, 95\%, confidence interval 2.4-7.6) of which heterozygous disease-associated variants are present in 9\% of CVID patients, compared to only monoallelic variants in $<2 \%$ of the healthy controls [17]. In healthy or mildly affected family members of CVID patients, some biallelic polymorphisms were found, suggesting that TACI variants may have an incomplete penetrance [17]. Furthermore, the presence of TACI polymorphisms in CVID predisposes to autoimmune disease and lymphoid hyperplasia [18].

Two TACI variants are significantly associated with CVID disease: C104R [17, 19] and A181E [19]. A characteristic of TNFRs is the presence of two cysteine-rich domain (CRDs) in the extracellular domain, which are conserved between species [20]. TACI monomers engage in a ligandindependent homotypic trimer formation that requires the CRD1 domain [21]. The second CRD allows ligation with BAFF or APRIL [22]. The amino acid substituting C104R variant is located in CRD2 and thus abrogates binding of APRIL and BAFF $[23,24]$ as was demonstrated in CVID patients heterozygous for C104R. Initially, the deficiency was attributed to a dominant-negative interference of the C104R polymorphism in trimeric complexes with TACI wildtype [21]. Subsequent reports; however, suggest haploinsufficiency of the TACI allele [25-27].

The A181E variant is located at the transmembrane region and permits normal ligand binding [23]. Mouse models with an A181E equivalent (A144E) show defective preligand and ligand-induced clustering of the intracellular domain and subsequent hampered nuclear factor kappa $\mathrm{B}(\mathrm{NF} \kappa \mathrm{B})$ activation; possibly, this is caused by a conformational change resulting from the negative charge introduced by the A181E variant [28]. A further consequence was low serum IgA levels and impaired antibody responses to type II T-cellindependent antigens.

To more closely resemble human conditions exhibiting heterozygous alleles, A181E and C104R were investigated in combination with a wildtype TACI allele. Neither variant interfered with oligomerization with wildtype TACI molecules nor $\mathrm{NF} \kappa \mathrm{B}$ activation was unaffected in $293 \mathrm{~T}$ cells with wildtype and mutant TACI [26]. Dominant-negative interference of the variants with wildtype TACI is therefore improbable, but haploinsufficiency was not completely excluded. It was therefore recommended to study B-cell function in patients, their healthy relatives, and unrelated subjects who carry the same mutation [26]. Our study here addressed this suggestion.

We identified a patient compound heterozygous for A181E and C104R. Her disease course was complicated by cytopenias, interstitial lung disease, autoimmune nephritis, and eventually mortality [29]. In contrast, her sibling carried an identical TACI genotype but was completely healthy, which was not reported previously. Here we describe a clinical and immunological evaluation of the patient compared to the healthy sibling and unrelated healthy and CVID controls. We provide mechanistic support of how individuals with identical TACI alleles may exhibit differential TACI protein expression and downstream signaling function.

\section{Materials and Methods}

2.1. Patients. As part of routine evaluation of CVID patients in our institute, C104R and A181E TACI variants were screened by polymerase chain reaction (PCR) as described previously [29] and both were found in the index patient. As we considered stem cell transplantation in the index patient, parents and siblings were screened (Figure 2(a)). Informed consent was obtained to further evaluate the patient and family members for clinical indications. Further data were obtained from medical records.

2.2. DNA Sequencing. TACI mutations were investigated as described previously [29]. Briefly, primers were designed for the p.C104R and p.A181E variants and their wildtypes with Primer Express software (Applied Biosystems). To test for additional mutations or splicing variants [22], primers were designed for the complete TACI sequence, containing 5 exons. The following primers were used: reverse $5^{\prime}$-TGTAAAACGACGGCCAGTCAGCCCAAGCACTAATCAAATC- $3^{\prime}$ and forward $5^{\prime}$-CAGGAAACAGCTATGACССТСТCTCСССТCСТCTCCATC- $3^{\prime}$. For splicing variants cDNA was sequenced using a 3730 DNA Analyzer. Prior to sequencing the TACI domain of the CDNA was amplified by a PCR starting at $95^{\circ} \mathrm{C}$ for $10 \mathrm{~min}$ followed by 35 cycles of $58^{\circ} \mathrm{C}$ for $15 \mathrm{~s}$ and $60^{\circ} \mathrm{C}$ for $1 \mathrm{~min}$.

2.3. Flow Cytometry. Phenotypic and functional evaluation of the lymphocyte compartment was performed as described previously [29]. Next, lymphoblastoid cell lines (LCL) were generated using Epstein-Barr virus and stained with antibodies against CD19, CD20, CD21 (all Becton Dickinson), TLR9, CD40 (both eBioscience), rabbit polyclonal anti-TACI (Abcam) with secondary anti-rabbit Ig-DiLight649 (Jackson Immunoresearch, West Grove, PA), and the appropriate isotypes. Intracellular TACI expression was determined after treatment with permeabilization reagents.

For calcium assays fresh peripheral blood mononuclear cells (PBMC) were incubated with Fluo-3, Fura Red (both Molecular Probes) (30 min), and CD19 (10 min) at $37^{\circ} \mathrm{C}$. Cells 
were washed twice and resuspended in Hank's balanced salt solution (HBSS) supplemented with $10 \%$ fetal calf serum (FCS). Cytosolic calcium levels were measured on Facs Canto II; after 1 minute $20 \mu \mathrm{g} / \mathrm{mL}$ goat anti-human IgM $\mathrm{F}\left(\mathrm{ab}^{\prime}\right)_{2}$ fragments (Jackson Immunoresearch) were added. After another 5 minutes $20 \mu \mathrm{g} / \mathrm{mL}$ ionomycin (Calbiochem, San Diego, CA) was added. Area under curves was calculated using Facs Diva and Prism software. Next, LCL were stained and resuspended in calcium-free phosphate-buffered saline (PBS). Calcium chloride was added after intracellular calcium storage depletion with ionomycin or thapsigargin (Santa Cruz Biotechnology, Santa Cruz, CA).

2.4. In Vitro Stimulation Assays and PCR. LCL were cultured in RPMI 1640 containing 10\% FCS at the subsequent conditions: mouse anti-TACI $(0.5 \mu \mathrm{g} / \mathrm{mL}, \mathrm{R} \& \mathrm{D}$ systems, Minneapolis, MN, USA) and goat anti-mouse IgG microbeads (Miltenyi Biotec, Carlsbad, CA) with or without IL-4 (100 U/mL, Immunotools); CpG phosphorothioatemodified oligodeoxynucleotide $2006(0.5 \mu \mathrm{g} / \mathrm{mL}$, Alexis Biochemicals), IL-10 (10 ng/mL, Becton Dickinson), or anti-CD40 (0.1 $\mu \mathrm{g} / \mathrm{mL}, \mathrm{R} \& \mathrm{D}$ systems). After 48 hours, cells were lysed, and total mRNA was isolated using Tripure isolation reagent (Roche Diagnostics, Mannheim, Germany) according to the manufacturer's instructions. Reverse transcription was performed using an iScript cDNA synthesis kit (Biorad, Hercules, CA). Primers were mixed with IQ SYBR green supermix (BioRad). The detection run started at $95^{\circ} \mathrm{C}$ for $10 \mathrm{~min}$, followed by 45 cycles of $95^{\circ} \mathrm{C}$ for $15 \mathrm{~s}$ and $60^{\circ} \mathrm{C}$ for $1 \mathrm{~min}$. Assays were performed in duplicate or triplicate as $15 \mu \mathrm{L}$ reactions in 96-well plates using C1000 Thermal Cycler (BioRad). Results were normalized to the endogenous GAPDH mRNA $\left(2^{-\Delta C T}\right)$. The following primers were used: GAPDH forward: 5'-GTCGGAGTCAACGGATT-3'; GAPDH reverse: $5^{\prime}$-AAGCTTCCCGTTCTCAG-3'; AICDA forward: $5^{\prime}$-TGCTCTTCCTCCGCTACATCTC-3'; AICDA reverse: $5^{\prime}$-AACCTCATACAGGGGCAAAACC- $3^{\prime}$; NF $\kappa$ B forward: $5^{\prime}$-GAAGCACGAATGACAGAGGC- ${ }^{\prime}$; NF $\kappa$ B reverse $5^{\prime}$-GCTTGGCGGATTAGCTCTTT-3 ${ }^{\prime}$; NFAT forward $5^{\prime}$-GCAGAGCACGGACAGCTATC- $3^{\prime}$; NFAT reverse $5^{\prime}$ GGGCTTTCTCCACGAAAATGA-3' (All Sigma-Aldrich).

\section{Results}

3.1. Clinical Evaluation of the CVID Index Patient. The index patient (Figure 2(a), arrow) was referred at age 11 for recurrent upper and lower respiratory tract infections and splenomegaly. Laboratory investigations showed absent isohemagglutinins and decreased serum titers of total IgG, $\operatorname{IgA}, \operatorname{Ig} M, \operatorname{IgG}_{1}$, and $\operatorname{IgG}_{2}$ (Table 1 ). She was lymphopenic, with low numbers of $\mathrm{B}$ cells, $\mathrm{CD}^{+}$, and $\mathrm{CD}^{+} \mathrm{T}$ cells and almost absent class switched memory B cells (Figure 1(a)). Functionally, in vitro $\mathrm{T}$-cell proliferation assays showed a positive response to mitogens and an incomplete response to particularly viral antigens. A diagnosis of CVID was made, and immunoglobulin replacement therapy was initiated.

After some time she developed CVID-related disease manifestations, including autoimmune hemolytic anemia
(AIHA), idiopathic thrombocytopenic purpura (ITP), submandibular lymphadenopathy, and autoimmune nephritis; a renal biopsy showed tubulo interstitial nephritis with granulomatous changes; the estimated glomerular filtrated rate was $41.2 \mathrm{~mL} / \mathrm{min} / 1.73 \mathrm{~m}^{2}$ (as calculated by Schwartz formula). Furthermore, she developed exercise-induced dyspnea; a high-resolution computed tomography (HRCT) scan showed airway and interstitial lung disease with bronchiectasis and peribronchovascular abnormalities, consisting of focal lesions with ground glass aspect, peribronchial consolidations, bronchial wall thickening, and air trapping (Figure 2(b)). A broncho alveolar lavage showed no indication for viral, bacterial, or fungal infections. Pulmonary findings were suggestive for granulomatous disease, and lowdose steroids and tacrolimus were initiated, resulting in normalization of pulmonary function and glomerular filtration rate. Furthermore, viral immunity appeared to be affected, as cytomegalovirus was repeatedly detectable with viral loads of 100-2000 copies/mL in plasma. Treatment with tacrolimus was complicated by tubulopathy, and therefore mycophenolate mofetil was started with good initial response, however, causing pancreatitis. Rituximab was administered, with positive effect on lymphadenopathy, AIHA, and ITP. Despite all treatment efforts taken, disease manifestations progressed further and became refractory. The index patient underwent allogeneic stem cell transplantation with matched unrelated cord blood after myeloablative conditioning, but eventually died of the complications of graft versus hose disease combined with multiple infections.

3.2. Clinical and Immunological Evaluation of Family Members. Further studies were performed to determine the role of these TACI variants. Clinically the parents and sibling 2 were healthy; sibling 1 developed clinical symptoms of CVID during the course of investigations. Serum Ig levels were normal in both parents and sibling 2, apart from a modestly decreased serum IgA. In contrast, sibling 1 was hypogammaglobulinemic for $\operatorname{IgG}, \operatorname{IgA}$, and subclasses $\operatorname{IgG}_{1}$, $\mathrm{IgG}_{2}$, and $\mathrm{IgG}_{4}$, albeit modestly in comparison to the index patient (Table 1).

To study specific antibody synthesis, parents and sibling 1 and 2 were vaccinated with 23-valent Pneumovax (Adventis Pasteur MSD), and total IgG antibody titers were measured after 1 month. Parents and sibling 2 had adequate T cellindependent responses in vivo to pneumococcal polysaccharides, while the response of sibling 1 was suboptimal (Table 1).

Consequently, immunological evaluation was extended in siblings 1 , revealing an increase in relative and absolute numbers of $\mathrm{CD}^{+} \mathrm{T}$ cells. Total $\mathrm{B}$ cells were increased (21.7\% of lymphocytes, 512 cells $/ \mu \mathrm{L})$, and the composition of B-cell compartment remained normal. Proliferative T-cell responses in vitro were normal to mitogens but partially impaired to recall antigens, similar to the index patient. Total B-cell count and composition of B-cell compartment of sibling 2 were normal (Figure 1(b)).

Sibling 1 slowly developed clinical symptoms, consisting of diarrhea, fatigue, and persistent rhinosinusitis refractory to antibiotic prophylaxis. The spleen was enlarged on abdominal 

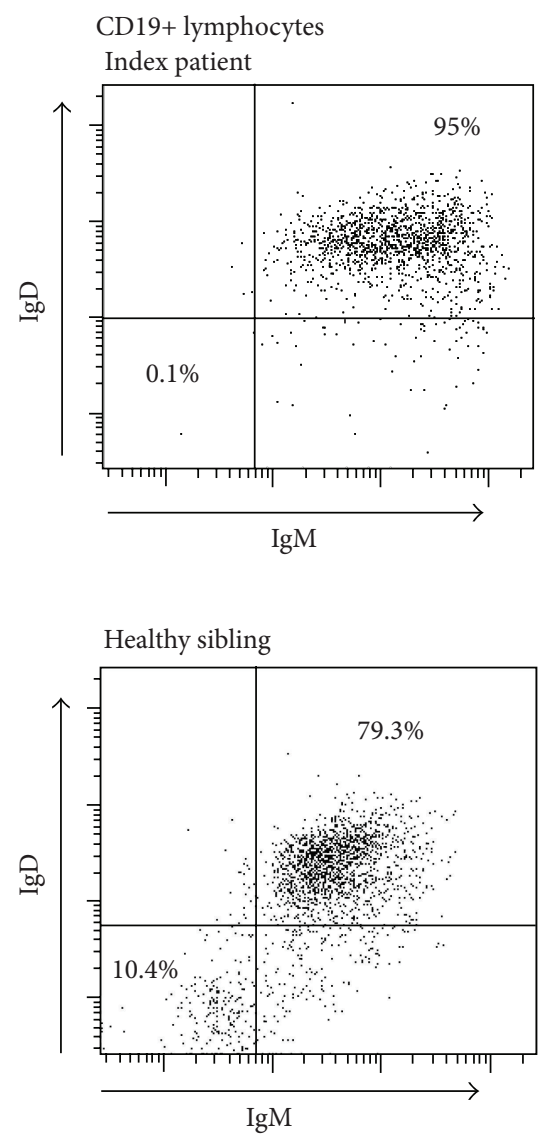

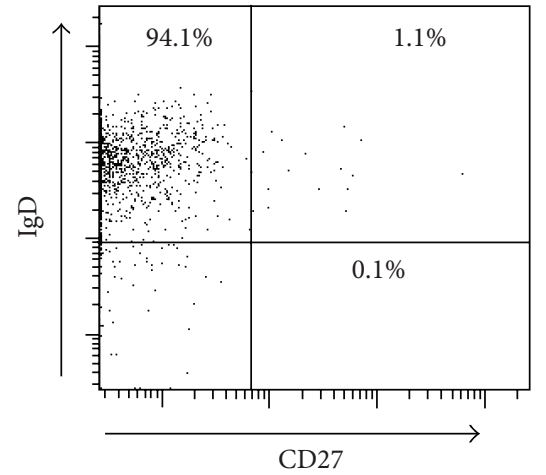

(a)

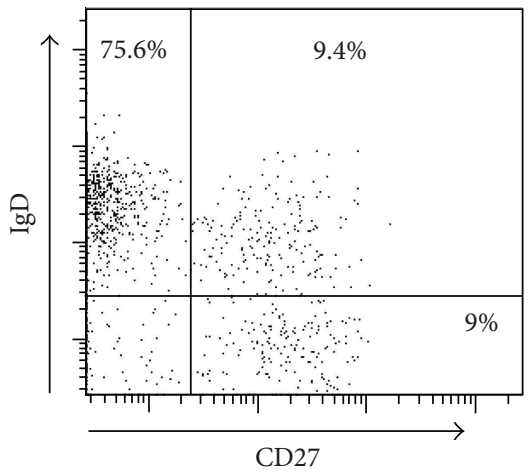

(b)
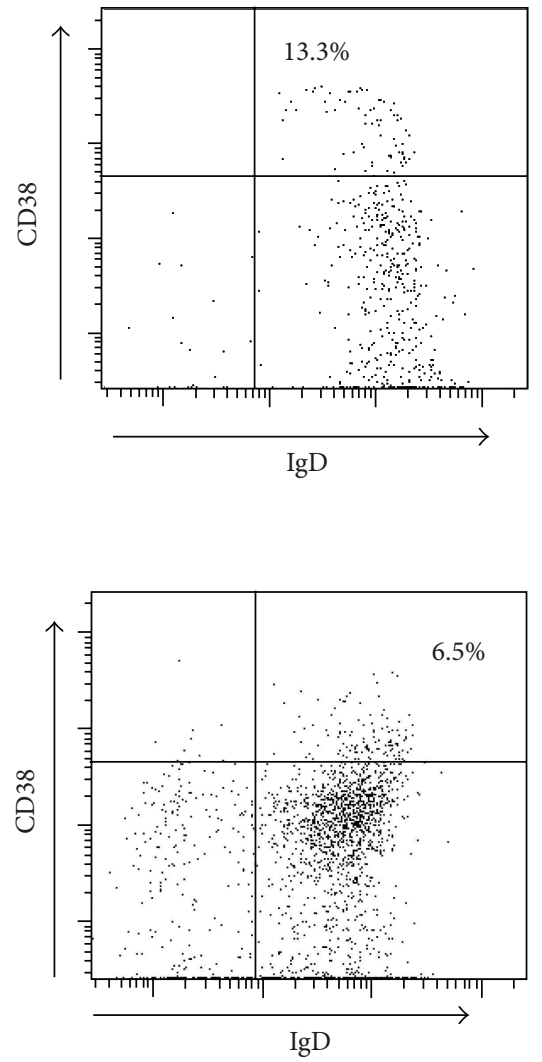

Figure 1: B-cell subpopulations of index patient and healthy sibling. (a) B cell subpopulations of index patient, showing $94.1 \%$ naïve + transitional B cells (IgM+IgD+CD27-), 1.1\% Marginal Zone B cells (IgM+IgD+CD27+), 0.1\% class-switched B cells (IgD-IgM-CD27+) and $13.3 \%$ transitional B cells (IgD+CD38+). (b) B cell subpopulations of healthy sibling, showing $75.6 \%$ naïve + transitional B cells (IgM+IgD+CD27-), 9.4\% Marginal Zone B cells (IgM+IgD+CD27+), 9\% class-switched B cells (IgD-IgM-CD27+), and 6.5\% transitional B cells $(\mathrm{IgD}+\mathrm{CD} 38+)$.

echography. Based on these clinical and laboratory findings, immunoglobulin replacement therapy was initiated, which led to significant clinical improvement. Further studies were focused on the index patient and asymptomatic sibling 2 .

\subsection{TNFRSF13B Compound Heterozygous Variants in the} Index Patient and Asymptomatic Sibling. TACI is a CVID susceptibility factor, and to substantiate the diagnosis CVID, we performed gene sequencing of the TACI gene. Analysis of the patient's TNFRSF13B gene showed two variants C104R and A181E. Subsequently, her family was investigated, revealing that each parent carried one variant (Figure 2(a)). The brother (sibling 3 ) had two normal TACI alleles and was not further investigated. The two sisters (siblings 1 and 2) were also compound heterozygous for A181E/C104R (Figure 2(a)). Sequencing of the complete TNFRSF13B gene revealed no additional mutations [22]. The index patient and sibling 2 exhibited identical alternative splicing patterns of exon 2 that encode the CRD1, important in trimer formation of TACI [22]. This finding, however, did not help explain the different clinical and B-cell activation phenotype between our index patient and sibling 2 .

3.4. TACI Protein Expression and Function in the Index Patient and Asymptomatic Sibling. Lymphoblastoid cell lines (LCL) were generated of the index patient and sibling 2 and compared to cell lines of healthy controls, CVID patients, and 2 CVID patients with a monoallelic A181E variant. LCL of all subjects exhibited TACI expression on the cell membrane. Interestingly, the TACI mean fluorescence intensity of the index patient was lower in comparison with the other subjects, especially with sibling 2 , from whom LCL repeatedly displayed the highest cell surface expression (Figure 2(c)). Subsequently, intracellular TACI was measured to study the possibility of impaired transition of TACI to the cell surface and ensuing intracellular TACI accumulation. Intracellular TACI, however, correlated with the levels of cell surface TACI (Pearson correlation $0.70, P=0.037$ ) and was also repeatedly lower in the index patient (Figure 2(c)). Basal TACI mRNA levels varied within a similar range for all LCL, including the index patient and sibling (Figure 2(d)). 


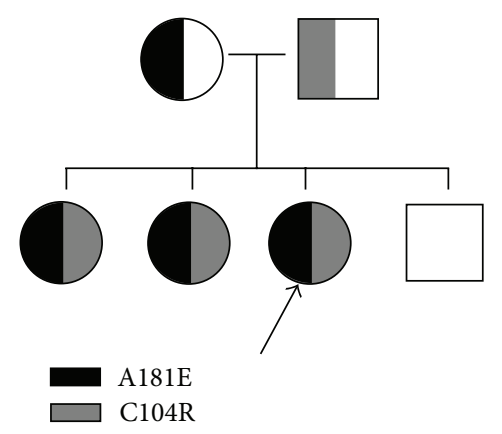

(a)

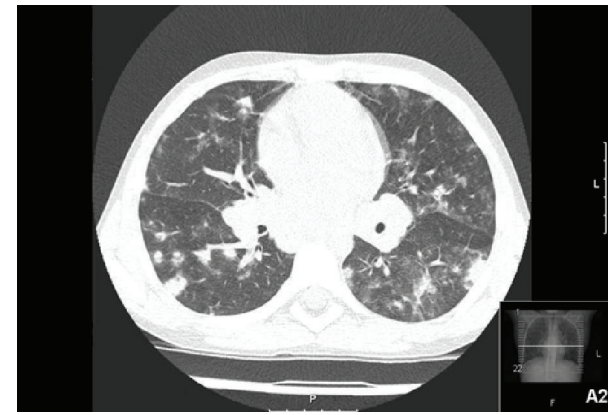

(b)
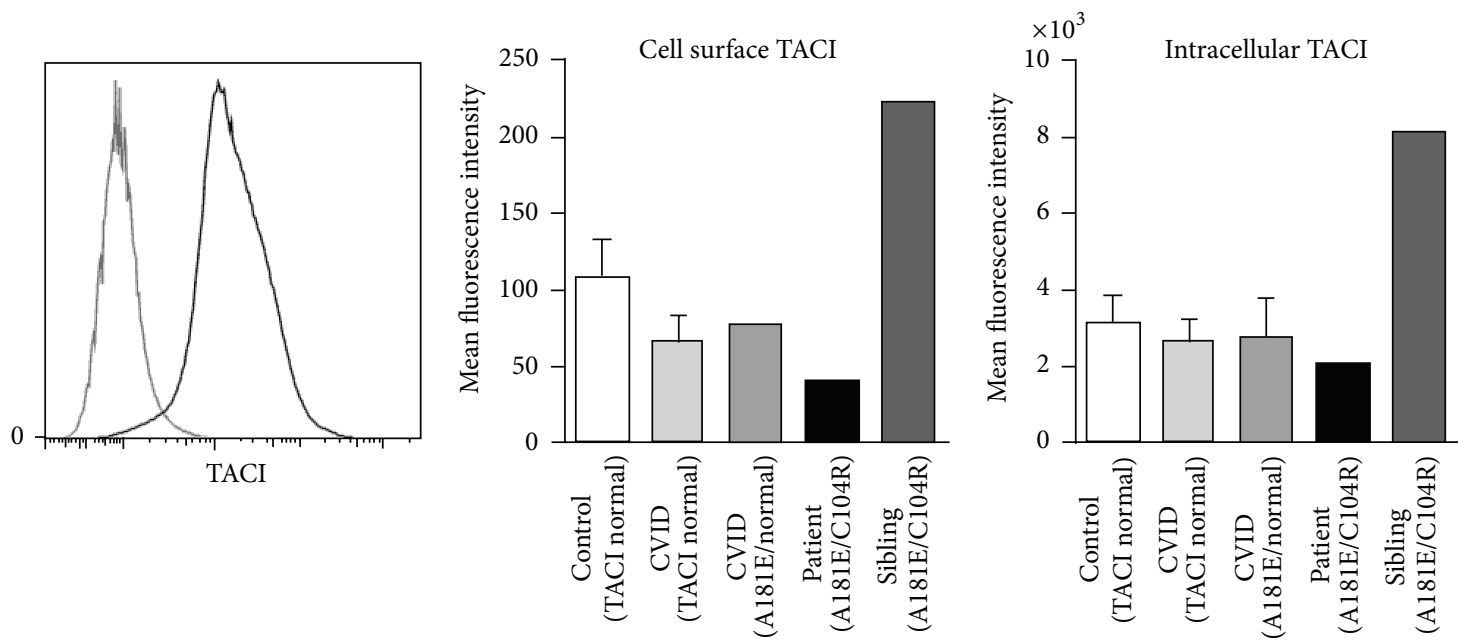

(c)

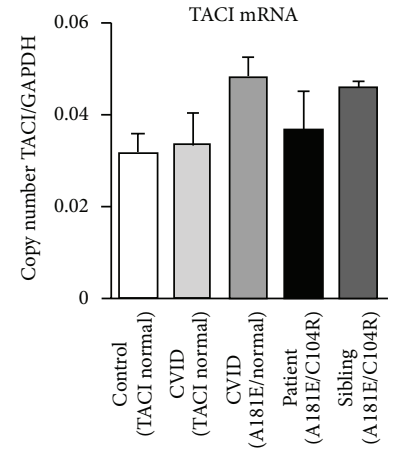

(d)
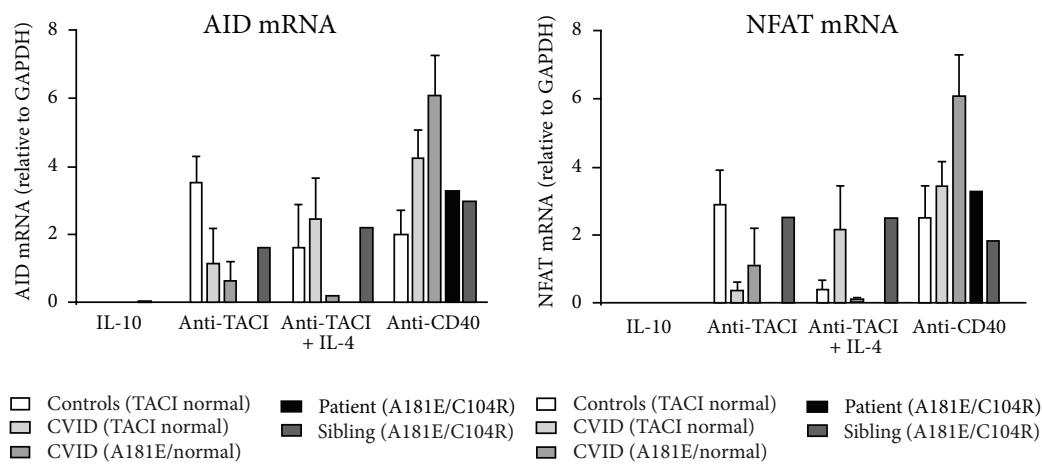

(e)

FIgURE 2: TACI expression and function are decreased in the CVID patient compared to her asymptomatic sibling. (a) Pedigree of the studied family. Circles display females; squares display males. The index patient is indicated with an arrow. (b) Chest high-resolution CT scan of the index patient, revealing peribronchial consolidations with ground glass appearance and peribronchial thickening. ((c)-(d)) TACI surface (c ii) or intracellular (c iii) protein and mRNA expression. Light grey line indicated isotype indicated dark grey, TACI (c i). Results of 5 healthy donors: 3 CVID patients with normal TACI, 2 CVID patients with monoallelic A181E, the index patient and sibling 2. For (d) average results of 3 independent experiments are shown. (e) AID (left) and NFAT (right) mRNA induction after $48 \mathrm{~h}$ culture of LCL with the indicated stimuli. Bars represent means \pm standard error of the mean.

Next, we investigated TACI function by measuring its ability to induce activation-induced cytidine deaminase $(A I C D A)$ gene transcription. The AICDA gene product activation-induced deaminase (AID) is an enzyme of pivotal importance for secondary antibody diversification [30]. Healthy control and CVID patient LCL with normal biallelic TACI showed AID mRNA induction upon stimulation with agonistic anti-TACI (Figure 2(e)). CVID patients with monoallelic A181E variants showed minimal AICDA gene transcription. The index patient had no AICDA gene transcription upon TACI stimulation, while AID mRNA levels of sibling 2 LCL were comparable to B cells with normal TACI. The same pattern was noticed for NFAT mRNA induction; gene transcription upon TACI triggering was lower in 
TABLE 1: Immunological characteristics of patient and family.

\begin{tabular}{|c|c|c|c|c|c|}
\hline & Patient & Sibling 1 & Sibling 2 & Mother & Father \\
\hline Gender & ㅇ & ㅇ & ㅇ & ㅇ & 0 \\
\hline Age (yrs) & 11 & 16 & 14 & 43 & 43 \\
\hline TACI variant & $\mathrm{C} 104 \mathrm{R}+\mathrm{A} 181 \mathrm{E}$ & $\mathrm{C} 104 \mathrm{R}+\mathrm{A} 181 \mathrm{E}$ & $\mathrm{C} 104 \mathrm{R}+\mathrm{A} 181 \mathrm{E}$ & $\mathrm{A} 181 \mathrm{E}$ & C104R \\
\hline Infections & Yes & Initially no, later yes & No & No & No \\
\hline $\operatorname{IgG}$ & $2.3 \downarrow$ & $4.87 \downarrow$ & 8.37 & 14.2 & 11.4 \\
\hline $\operatorname{Ig} A$ & $0.12 \downarrow$ & $0.36 \downarrow$ & $0.45 \downarrow$ & 1.7 & 1.1 \\
\hline IgM & $0.20 \downarrow$ & $0.59 \downarrow$ & 0.92 & 1.4 & 0.78 \\
\hline IgG1 & $1.5 \downarrow$ & $3.3 \downarrow$ & 5.7 & $9.8 \uparrow$ & 7.6 \\
\hline IgG2 & $0.11 \downarrow$ & $0.5 \downarrow$ & 1.27 & 3.36 & 2.08 \\
\hline IgG3 & $1.66 \uparrow$ & 0.52 & 0.54 & 1.00 & 0.87 \\
\hline IgG4 & $<0.07$ & $0.04 \downarrow$ & 0.18 & 0.05 & 0.24 \\
\hline Specific Ab titer after vaccination pneumococcal serotype & $(\mathrm{IU} / \mathrm{mL})$ & $(\mu \mathrm{g} / \mathrm{mL})$ & $(\mu \mathrm{g} / \mathrm{mL})$ & $(\mu \mathrm{g} / \mathrm{mL})$ & $(\mu \mathrm{g} / \mathrm{mL})$ \\
\hline 1 & & 1.4 & 7.2 & 15 & $>40$ \\
\hline 3 & $1 \downarrow$ & 1.2 & 6.1 & 7.0 & 2.6 \\
\hline 4 & $2 \downarrow$ & $0.46 \downarrow$ & 6.2 & $0.89 \downarrow$ & 1 \\
\hline 5 & & 4.0 & $0.34 \downarrow$ & 2.4 & 6.9 \\
\hline $6 \mathrm{~B}$ & & $0.050 \downarrow$ & 1.5 & 11 & $0.65 \downarrow$ \\
\hline $7 \mathrm{~F}$ & & 1.7 & 19 & 1.2 & 15 \\
\hline $9 \mathrm{~V}$ & $2 \downarrow$ & $0.97 \downarrow$ & 6.6 & 6.9 & 4.5 \\
\hline 14 & & 3.1 & $>40$ & 11 & 39 \\
\hline $18 \mathrm{C}$ & & $0.49 \downarrow$ & $0.70 \downarrow$ & 3.7 & 5.2 \\
\hline $19 \mathrm{~F}$ & & $0.48 \downarrow$ & $>40$ & 39 & 12 \\
\hline $23 \mathrm{~F}$ & & 1.8 & 1.4 & 23 & 11 \\
\hline Anti-Hib IgG & 31 & $>100$ & $>100$ & 8.7 & 1.6 \\
\hline
\end{tabular}

Numbers with $\uparrow$ indicate values $\geq 2$ standard deviations (SD) above the references values; $\downarrow$ are numbers $\leq 2$ SD age-related references values. $\mathrm{Ab}$ : antibody; Ig: immunoglobulin; TACI: transmembrane activator, calcium modulator, and cyclophilin ligand interactor; Hib: Haemophilus influenzae type B.

the index patient compared to sibling 2. There were no differences in $\mathrm{NF} \kappa \mathrm{B}$ induction upon TACI stimulation. Stimulation of the CD40 pathway showed adequate induction of AID and NFAT mRNA in the index patient. IL-10 treatment was included as negative control that does not induce AICDA gene expression. Taken together, TACI protein expression and function were increased in sibling 2 when compared to the index patient.

3.5. Appearance of TLR9 Protein Expression and Function in the Asymptomatic Sibling. We considered two possibilities that may explain why the index patient and sibling 2 , having identical TACI gene mutations, exhibit divergent health consequences: either the index patient had an additional immune defect, which in combination with the TACI variants would lead to CVID disease, or sibling 2 has compensatory immune function in the TACI signaling route that prevents CVID disease from occurring. First, as defects in the Bcell receptor (BCR) and its coreceptor complex have been described [31, 32], we screened the BCR signalosome. CD19, CD21, and CD20 cell surface expression of the index patient were comparable to expression on control LCL (data not shown). Functionally, early B-cell activation seemed intact, as measured by calcium mobilization after BCR stimulation of fresh PBMC (Figure 3(a)). The index patient showed a normal initial but rapidly declining calcium influx, as previously observed in CVID patients with autoimmune symptoms (own unpublished observations), and area under curves were comparable to controls. As the index patient's endoplasmic reticulum (ER) calcium storage capacity was not decreased compared to sibling 2, an additional defect in BCR signaling was considered improbable.

Second, the classical route of BCR isotype class switching is via CD40 with CD40L on T cells, and it was recently shown that TACI and CD40 signaling can act in synergy $[13,15]$. However, CD40 cell surface expression (Figure 3(b)) and CD40-mediated gene transcription (Figures 2(e) and 3(b)) were comparable between all LCL suggesting that CD40 signaling in the index patient B cells was intact, at least in this assay.

Third, we explored TLR9 signaling, as TLR9 defects have been described in CVID [2,3], and TLR9 and TACI signaling employ common downstream signaling pathways $[15,16]$. Noticeably, TLR9 expression was nearly twofold higher in sibling 2 compared to other LCL, and this increased expression was accompanied by an increased TLR9 expression and function (Figure 3(c)), supporting the possibility that compensatory TLR9 expression contributes as a mechanism for altered TACI A181E/C104R protein, in restoring B-cell overall capacity to transmit signals towards NFAT and AID. Thus, 


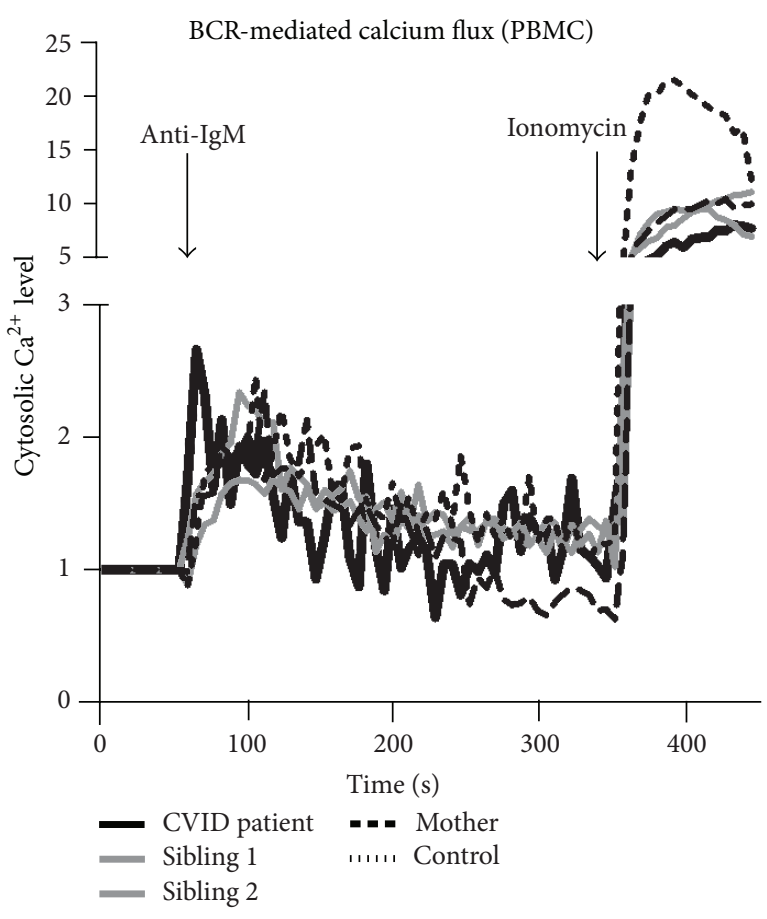

(a)

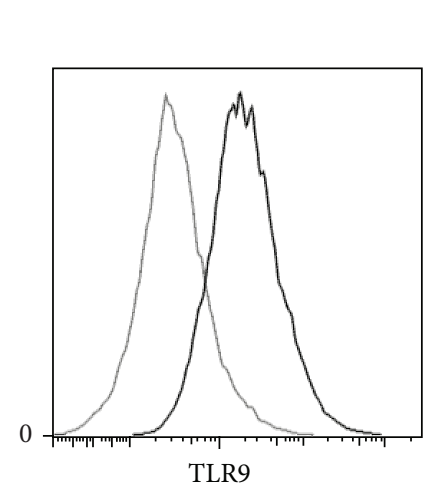

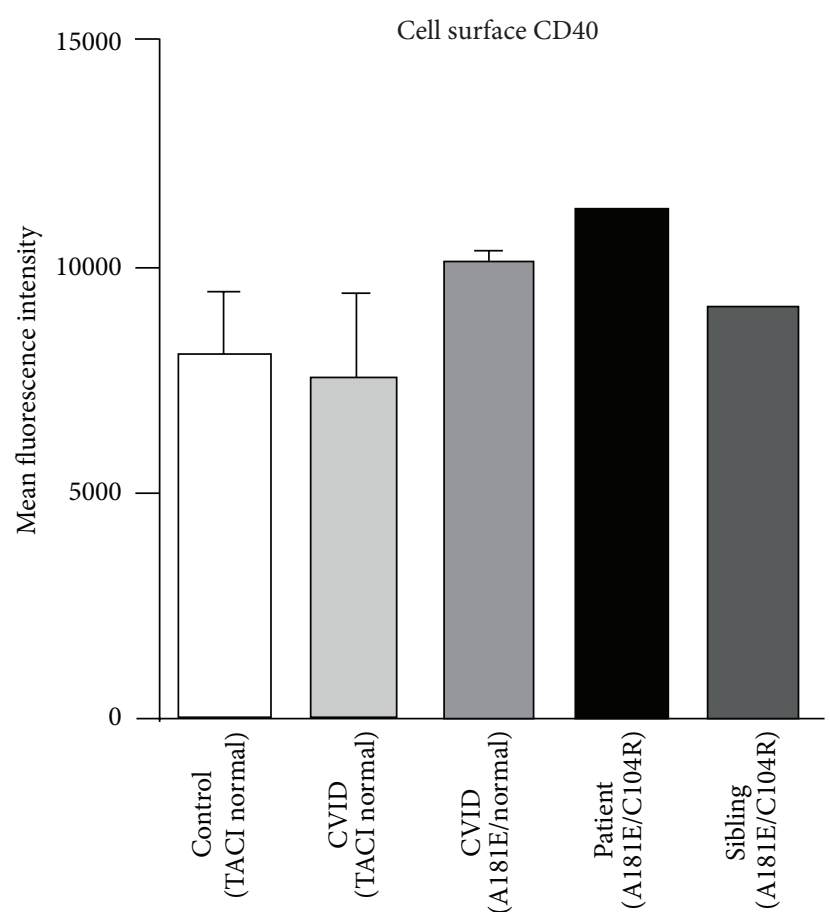

(b)
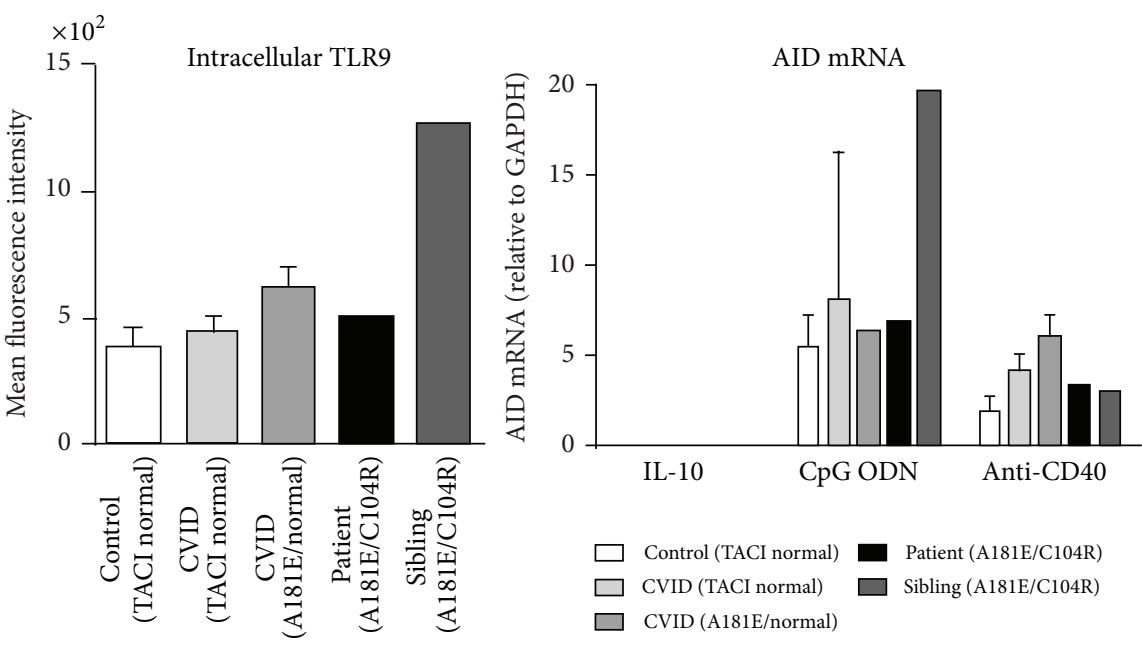

(c)

FIGURE 3: Similar BCR signaling but possibly enhanced TLR9 signaling in the healthy sibling. (a) Calcium mobilization upon triggering of the BCR using IgM F $\left(\mathrm{ab}^{\prime}\right)_{2}$ fragments in freshly isolated PBMCs. (b) CD40 surface expression as measured by flow cytometry. (c) TLR9 B-cell expression (c ii) and function (c iii). Light grey line indicated isotype indicated dark grey, TLR9 (c i). Bars represent means \pm standard error of the mean.

our data supports the notion that compensatory signaling in the TACI route, in the case of sibling 2 via enhanced TLR9 signaling, may prevent the development of CVID disease.

\section{Discussion}

We here describe differential immunological phenotypes displayed by siblings with identical genetic TACI variants. This family provides the unique opportunity to study the role and significance of the human C104R and A181E variants both in vivo and in vitro.

The index patient had severe CVID, which gradually evolved to late onset combined immunodeficiency, a phenomenon previously described in CVID patients [33]. Sibling 1 did eventually develop mild CVID, but without autoimmune manifestations. To date, sibling 2 remains free of symptoms and has excellent specific antibody responses, nevertheless this sibling may still develop CVID at a later age. Previous studies have shown that C104R abrogates ligand 
binding due to haploinsufficiency, whereas in the A181E variant, ligand binding remains intact $[21,26]$. At least a proportion of A181E/C104R TACI trimers will thus be able to bind ligand. As $\mathrm{NF} \kappa \mathrm{B}$ activation was strongly decreased in both variants, we hypothesized TACI function to be affected. AID and NFAT mRNA induction were reduced in the index patient and seemed also reduced in the CVID patients with a heterozygous A181E variant [14]. Conversely, sibling 2 showed AID and NFAT induction comparable to healthy controls and CVID patients with unaffected TACI alleles. NFAT activation induces transcription of cytokine genes encoding, for example, IL-4, which is involved in class switch recombination. IL-4 and CD40 ligation induces AID mRNA and protein expression and depends on STAT6 and $\mathrm{NF} \kappa \mathrm{B}$ [34]. AID is pivotal for class switch recombination and somatic hypermutation of the BCR $[35,36]$; both mechanisms are routinely impaired in CVID.

The differential TACI function in the index patient and sibling was accompanied by a change in TACI protein levels, showing increased TACI expression in the sibling both at the cell surface and intracellular level. TACI protein levels in the healthy sibling were increased in comparison to healthy controls as well. As TNFRSF13B gene transcription was comparable between all subjects except for the healthy sibling, these observations suggest that protein turnover in the healthy sibling may be decreased, possibly due to alterations in posttranslational modification of TACI protein, affecting protein stability and folding, transport, or degradation.

Alternatively, altered TACI function in the healthy sibling was compensated by increasing the TACI protein quantity through cross-talk with related immune pathways. CVID is generally assumed to be a polygenetic disorder, and it is plausible that alterations in the index patient were not limited to the TNFRSF13B gene. This prompted us to analyze other essential immune pathways in B lymphocytes, and we investigated CD40, BCR and TLR9 signaling. We did not find any additional defect in BCR, or CD40 signaling. Nevertheless, for both pathways, defective interaction with other cells or factors, such as abrogated CD40L ligation on $\mathrm{T}$ cells or CD21- complement C3d fragment interactions, cannot be excluded.

In contrast, TLR9 expression and function were considerably dissimilar between index patient and sibling 2 . Since the TLR9 expression levels and function were comparable between the index patient and the other subjects but increased in sibling 2, we hypothesize that compensatory TLR9 expression occurs in the sibling. TLR9 recognizes unmethylated CpG-motif-containing bacterial or viral DNA $[37,38]$ and has a critical role in the prevention of several bacterial and viral infections. Ligation of TLR9 induces recruitment of MyD88 [30, 39], initiating several signaling events which eventually result in activation of $\mathrm{NF} \kappa \mathrm{B}$ and $\mathrm{AP}-$ 1 [40]. By the same token, increased TACI protein stability may participate in a compensatory mechanism in the healthy sibling, possibly related to TLR9 signaling. TLR9 engagement induced TACI upregulation and enhanced TACI-mediated Ig class switching of effector B cells resulting in increased IgG secretion in cells exposed to anti-TACI and IL-10 and induced secretion of $\operatorname{IgA}$.
This study has some limitations. The TLR9 signaling pathway through MyD88 could be studied more extensively, not only on a functional level but also through whole exome sequencing. The effects of TACI malfunctioning on expression and function of several other targets could have been addressed, including other TNF receptors and their ligands or signaling proteins downstream of TACI such as TRAFs, CAML, and AP-1. Additional defects may not be limited to B-cell lymphocytes; TACI expression can be induced in $\mathrm{CD}^{+}$and CD8 $\mathrm{T}^{+}$cells $[4,41]$; study of TACI-related T-cell function would be particularly interesting since the patient demonstrated chronic viral infections in vivo and impaired antigenic T-cells responses to several viruses in vitro.

\section{Conclusion}

We here describe the diverse clinical and immunological characteristics in siblings with an identical TACI genotype. These clinical differences are supported by differences in TACI protein expression and function. We propose that compensatory regulatory mechanisms involving posttranslational modification of TACI protein and TLR9 exist and may overcome the altered function of TACI trimers in asymptomatic TACI A181E/C104R individuals. Additional studies of TACI protein homeostasis, the TACI signaling pathway and interaction with TLR9, and expression and function of TLR9 downstream signaling molecules are therefore advisable.

\section{Abbreviations}

AICDA: Activation-induced cytidine deaminase

AID: $\quad$ Activation-induced deaminase

AIHA: Autoimmune hemolytic anemia

APRIL: A proliferation-inducing ligand

BAFF: B-cell activating factor

BCMA: B-cell maturation antigen

CAML: Calcium modulator and cyclophilin ligand

CRD: $\quad$ Cysteine-rich domain

CVID: Common variable immunodeficiency

ER: $\quad$ Endoplasmic reticulum

FCS: $\quad$ Fetal calf serum

HRCT: High-resolution computed tomography

ICOS: Inducible costimulator

Ig: Immunoglobulin

iNKT cells: Invariant natural killer T cells

ITP: Idiopathic thrombocytopenic purpura

IVIG: Intravenous immunoglobulin replacement therapy

LCL: Lymphoblastoid cell line

MyD88: $\quad$ Myeloid differentiation primary response gene 88

NFAT: Nuclear factor of activated T cells

NFאB: $\quad$ Nuclear factor kappa B

ODN: Oligodeoxynucleotides

PBMC: $\quad$ Peripheral blood mononuclear cells

PCR: $\quad$ Polymerase chain reaction 
PPD: Purified protein derivative

TACI: Transmembrane activator and CAML interactor

TLR: Toll-like receptor

TNFR: Tumor necrosis factor receptor

TRAF: TNFR-associated factor.

\section{Authors' Contribution}

Joris M. van Montfrans and Marianne Boes contributed equally.

\section{Acknowledgments}

The authors would like to thank the technicians of the Department of Medical Immunology for excellent assistance with the TACI sequencing setup and Ewoud Compeer and Arie Jan Stoppelenburg for help with PCR and data analyses.

\section{References}

[1] M. A. Park, J. T. Li, J. B. Hagan, D. E. Maddox, and R. S. Abraham, "Common variable immunodeficiency: a new look at an old disease," The Lancet, vol. 372, no. 9637, pp. 489-502, 2008.

[2] C. Cunningham-Rundles, L. Radigan, A. K. Knight, L. Zhang, L. Bauer, and A. Nakazawa, "TLR9 activation is defective in common variable immune deficiency," Journal of Immunology, vol. 176, no. 3, pp. 1978-1987, 2006.

[3] J. E. Yu, A. K. Knight, L. Radigan et al., "Toll-like receptor 7 and 9 defects in common variable immunodeficiency," Journal of Allergy and Clinical Immunology, vol. 124, no. 2, pp. 349-356, 2009.

[4] G.-U. Von Bülow and R. J. Bram, "NF-AT activation induced by a CAML-interacting member of the tumor necrosis factor receptor superfamily," Science, vol. 278, no. 5335, pp. 138-141, 1997.

[5] P. Schneider, F. Mackay, V. Steiner et al., "BAFF, a novel ligand of the tumor necrosis factor family, stimulates B cell growth," Journal of Experimental Medicine, vol. 189, no. 11, pp. 1747-1756, 1999.

[6] Y. Wu, D. Bressette, J. A. Carrell et al., “Tumor necrosis factor (TNF) receptor superfamily member TACI is a high affinity receptor for TNF family members APRIL and BLyS," Journal of Biological Chemistry, vol. 275, no. 45, pp. 35478-35485, 2000.

[7] S. A. Marsters, M. Yan, R. M. Pitti, P. E. Haas, V. M. Dixit, and A. Ashkenazi, "Interaction of the TNF homologues BLyS and APRIL with the TNF receptor homologues BCMA and TACI," Current Biology, vol. 10, no. 13, pp. 785-788, 2000.

[8] D. Bischof, S. F. Elsawa, G. Mantchev et al., "Selective activation of TACI by syndecan-2," Blood, vol. 107, no. 8, pp. 3235-3242, 2006.

[9] F. Mackay, P. Schneider, P. Rennert, and J. Browning, "BAFF and APRIL: a tutorial on B cell survival," Annual Review of Immunology, vol. 21, pp. 231-264, 2003.

[10] Y. Laabi, M. P. Gras, F. Carbonnel et al., "A new gene, BCM, on chromosome 16 is fused to the interleukin 2 gene by a $\mathrm{t}(4 ; 16)$ (q26;p13) translocation in a malignant $\mathrm{T}$ cell lymphoma," EMBO Journal, vol. 11, no. 11, pp. 3897-3904, 1992.
[11] J. S. Thompson, S. A. Bixler, F. Qian et al., "BAFF-R, a newly identified TNF receptor that specifically interacts with BAFF," Science, vol. 293, no. 5537, pp. 2108-2111, 2001.

[12] J. Hauer, S. Püschner, P. Ramakrishnan et al., “TNF receptor (TNFR)-associated factor (TRAF) 3 serves as an inhibitor of TRAF2/5-mediated activation of the noncanonical NF- $\kappa \mathrm{B}$ pathway by TRAF-binding TNFRs," Proceedings of the National Academy of Sciences of the United States of America, vol. 102, no. 8, pp. 2874-2879, 2005.

[13] E. Castigli, S. A. Wilson, A. Elkhal, E. Ozcan, L. Garibyan, and R. S. Geha, "Transmembrane activator and calcium modulator and cyclophilin ligand interactor enhances CD40-driven plasma cell differentiation," Journal of Allergy and Clinical Immunology, vol. 120, no. 4, pp. 885-891, 2007.

[14] E. Ozcan, L. Garibyan, J. J.-Y. Lee, R. J. Bram, K.-P. Lam, and R. S. Geha, "Transmembrane activator, calcium modulator, and cyclophilin ligand interactor drives plasma cell differentiation in LPS-activated B cells," Journal of Allergy and Clinical Immunology, vol. 123, no. 6, pp. 1277-1286, 2009.

[15] E. Ozcan, I. Rauter, L. Garibyan, S. R. Dillon, and R. S. Geha, "Toll-like receptor 9, transmembrane activator and calciummodulating cyclophilin ligand interactor, and CD40 synergize in causing B-cell activation," Journal of Allergy and Clinical Immunology, vol. 128, no. 3, pp. 601-609, 2011.

[16] B. He, R. Santamaria, W. Xu et al., "The transmembrane activator TACI triggers immunoglobulin class switching by activating B cells through the adaptor MyD88," Nature Immunology, vol. 11, no. 9, pp. 836-845, 2010.

[17] U. Salzer, C. Bacchelli, S. Buckridge et al., "Relevance of biallelic versus monoallelic TNFRSF13B mutations in distinguishing disease-causing from risk-increasing TNFRSF13B variants in antibody deficiency syndromes," Blood, vol. 113, no. 9, pp. 19671976, 2009.

[18] L. Zhang, L. Radigan, U. Salzer et al., "Transmembrane activator and calcium-modulating cyclophilin ligand interactor mutations in common variable immunodeficiency: clinical and immunologic outcomes in heterozygotes," Journal of Allergy and Clinical Immunology, vol. 120, no. 5, pp. 1178-1185, 2007.

[19] Q. Pan-Hammarström, U. Salzer, L. Du et al., "Reexamining the role of TACI coding variants in common variable immunodeficiency and selective IgA deficiency," Nature Genetics, vol. 39, no. 4, pp. 429-430, 2007.

[20] R. M. Locksley, N. Killeen, and M. J. Lenardo, "The TNF and TNF receptor superfamilies: integrating mammalian biology," Cell, vol. 104, no. 4, pp. 487-501, 2001.

[21] L. Garibyan, A. A. Lobito, R. M. Siegel, M. E. Call, K. W. Wucherpfennig, and R. S. Geha, "Dominant-negative effect of the heterozygous C104R TACI mutation in common variable immunodeficiency (CVID)," Journal of Clinical Investigation, vol. 117, no. 6, pp. 1550-1557, 2007.

[22] S. G. Hymowitz, D. R. Patel, H. J. A. Wallweber et al., "Structures of APRIL-receptor complexes: like BCMA, TACI employs only a single cysteine-rich domain for high affinity ligand binding," Journal of Biological Chemistry, vol. 280, no. 8, pp. 7218-7227, 2005.

[23] E. Castigli, S. A. Wilson, L. Garibyan et al., "TACI is mutant in common variable immunodeficiency and IgA deficiency," Nature Genetics, vol. 37, no. 8, pp. 829-834, 2005.

[24] U. Salzer, H. M. Chapel, A. D. B. Webster et al., "Mutations in TNFRSF13B encoding TACI are associated with common variable immunodeficiency in humans," Nature Genetics, vol. 37, no. 8, pp. 820-828, 2005. 
[25] C. Bacchelli, K. F. Buckland, S. Buckridge et al., "The C76R transmembrane activator and calcium modulator cyclophilin ligand interactor mutation disrupts antibody production and B-cell homeostasis in heterozygous and homozygous mice," Journal of Allergy and Clinical Immunology, vol. 127, no. 5, pp. 1253-1259, 2011.

[26] A. J. Fried, I. Rauter, S. R. Dillon, H. H. Jabara, and R. S. Geha, "Functional analysis of transmembrane activator and calciummodulating cyclophilin ligand interactor (TACI) mutations associated with common variable immunodeficiency," Journal of Allergy and Clinical Immunology, vol. 128, no. 1, pp. 226-228, 2011.

[27] J. J. Lee, H. H. Jabara, L. Garibyan et al., “The C104R mutant impairs the function of transmembrane activator and calcium modulator and cyclophilin ligand interactor (TACI) through haploinsufficiency," Journal of Allergy and Clinical Immunology, vol. 126, no. 6, pp. 1234-e2, 2010.

[28] J. J. Lee, I. Rauter, L. Garibyan et al., "The murine equivalent of the A181E TACI mutation associated with common variable immunodeficiency severely impairs B-cell function," Blood, vol. 114, no. 11, pp. 2254-2262, 2009.

[29] A. A. J. M. van de Ven, L. van de Corput, C. M. van Tilburg et al., "Lymphocyte characteristics in children with common variable immunodeficiency," Clinical Immunology, vol. 135, no. 1, pp. 6371, 2010.

[30] E. Latz, A. Schoenemeyer, A. Visintin et al., "TLR9 signals after translocating from the ER to CpG DNA in the lysosome," Nature Immunology, vol. 5, no. 2, pp. 190-198, 2004.

[31] C. Foerster, N. Voelxen, M. Rakhmanov et al., "B cell receptormediated calcium signaling is impaired in B lymphocytes of type Ia patients with common variable immunodeficiency," Journal of Immunology, vol. 184, no. 12, pp. 7305-7313, 2010.

[32] M. C. Van Zelm, J. Smet, B. Adams et al., "CD81 gene defect in humans disrupts CD19 complex formation and leads to antibody deficiency," Journal of Clinical Investigation, vol. 120, no. 4, pp. 1265-1274, 2010.

[33] M. Malphettes, L. Gérard, M. Carmagnat et al., "Late-onset combined immune deficiency: a subset of common variable immunodeficiency with severe T cell defect," Clinical Infectious Diseases, vol. 49, no. 9, pp. 1329-1338, 2009.

[34] F. Dedeoglu, B. Horwitz, J. Chaudhuri, F. W. Alt, and R. S. Geha, "Induction of activation-induced cytidine deaminase gene expression by IL-4 and CD40 ligation is dependent on STAT6 and NFאB," International Immunology, vol. 16, no. 3, pp. 395-404, 2004

[35] M. Muramatsu, K. Kinoshita, S. Fagarasan, S. Yamada, Y. Shinkai, and T. Honjo, "Class switch recombination and hypermutation require activation-induced cytidine deaminase (AID), a potential RNA editing enzyme," Cell, vol. 102, no. 5, pp. 553-563, 2000.

[36] P. Revy, T. Muto, Y. Levy et al., "Activation-induced cytidine deaminase (AID) deficiency causes the autosomal recessive form of the hyper-IgM syndrome (HIGM2)," Cell, vol. 102, no. 5, pp. 565-575, 2000.

[37] A. M. Krieg, "CpG motifs in bacterial DNA and their immune effects," Annual Review of Immunology, vol. 20, pp. 709-760, 2002.

[38] T. Kawai and S. Akira, "Innate immune recognition of viral infection," Nature Immunology, vol. 7, no. 2, pp. 131-137, 2006.

[39] E. Latz, A. Verma, A. Visintin et al., "Ligand-induced conformational changes allosterically activate Toll-like receptor 9," Nature Immunology, vol. 8, no. 7, pp. 772-779, 2007.
[40] S. Sato, H. Sanjo, K. Takeda et al., "Essential function for the kinase TAK1 in innate and adaptive immune responses," Nature Immunology, vol. 6, no. 11, pp. 1087-1095, 2005.

[41] F. Mackay and H. Leung, "The role of the BAFF/APRIL system on T cell function," Seminars in Immunology, vol. 18, no. 5, pp. 284-289, 2006. 


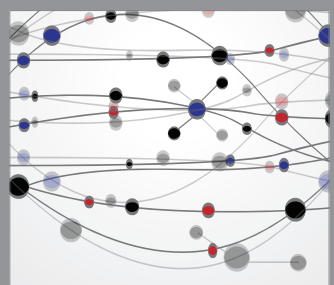

The Scientific World Journal
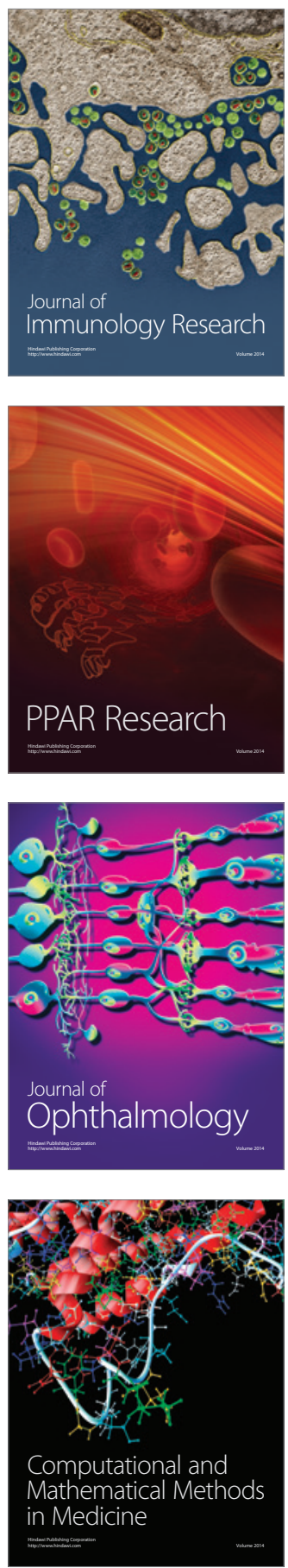

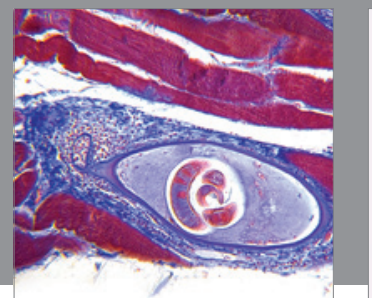

Gastroenterology

Research and Practice
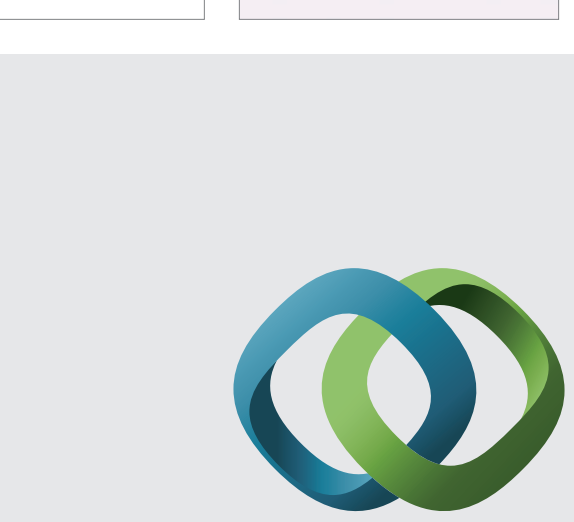

\section{Hindawi}

Submit your manuscripts at

http://www.hindawi.com
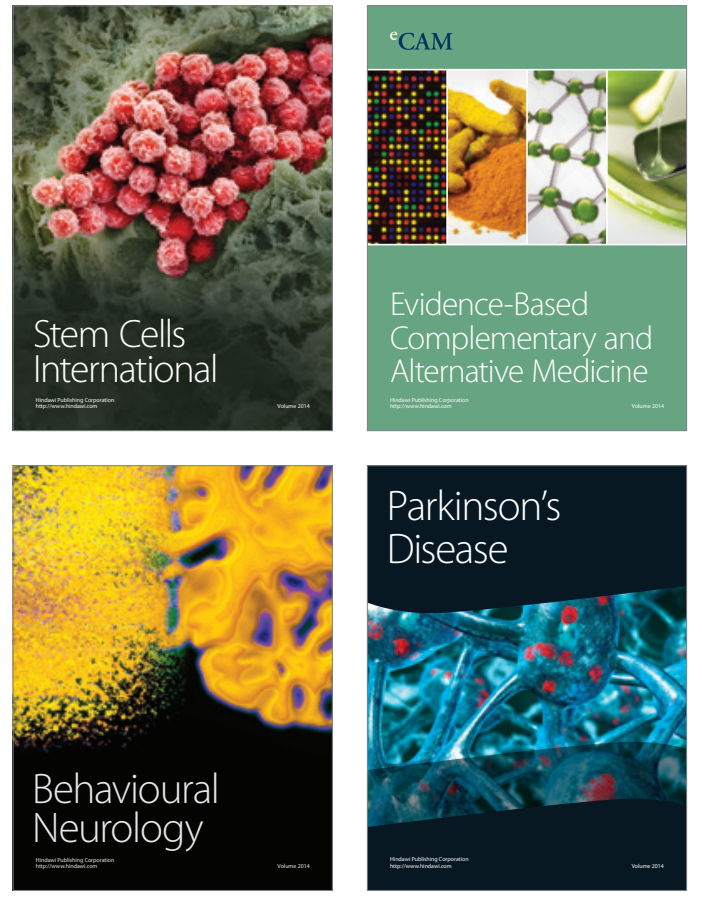
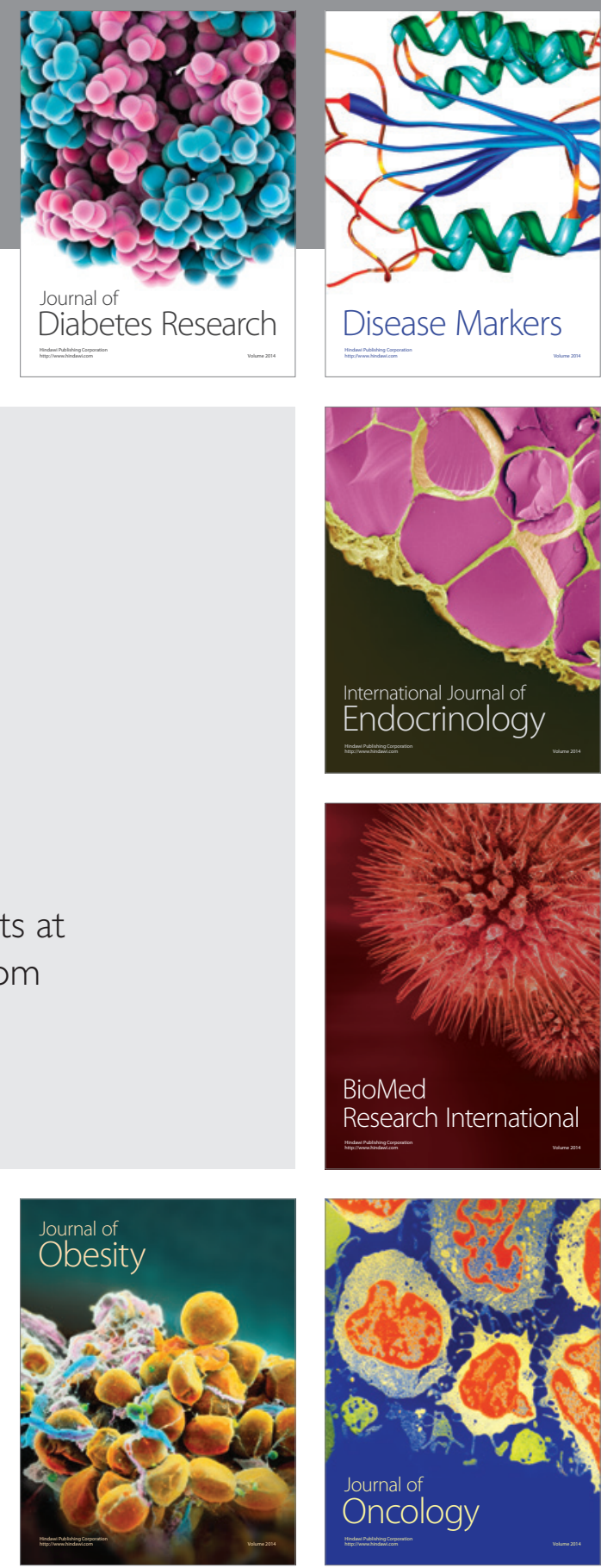

Disease Markers
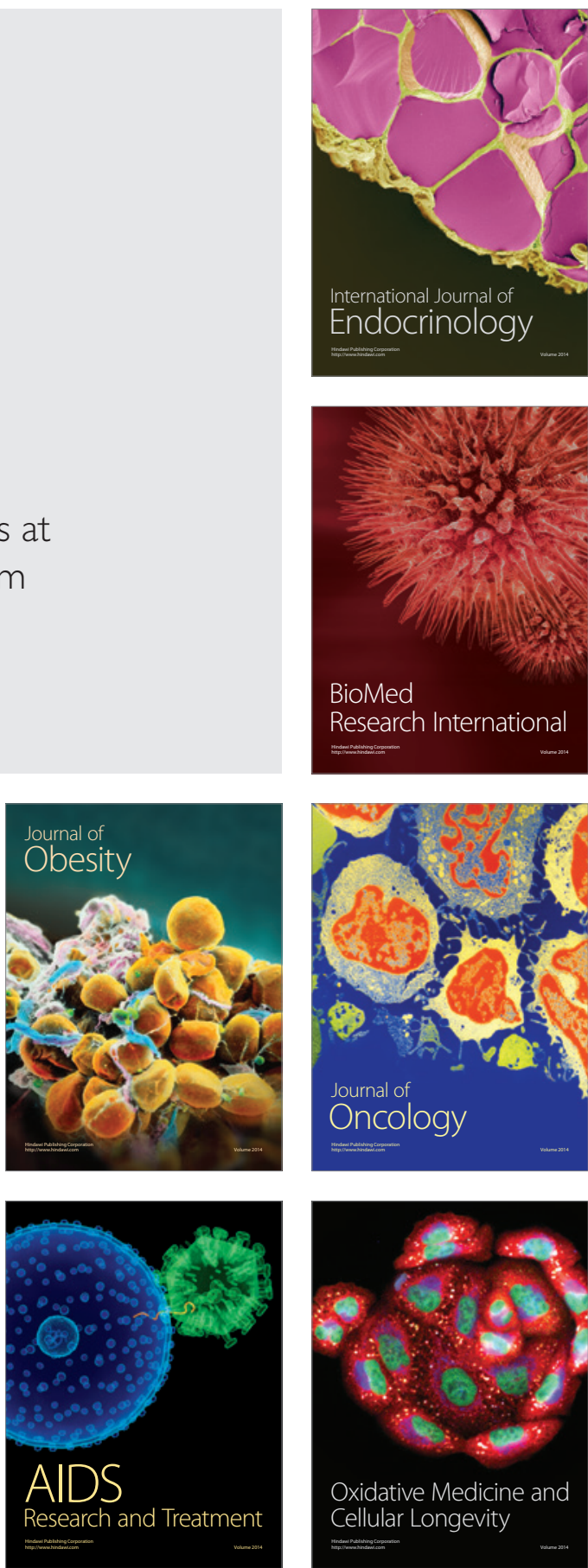